\title{
Framework Flexibility of ZIF-8 Under Liquid Intrusion: Discovering Time-Dependent Mechanical Response and Structural Relaxation
}

\author{
Yueting Sun, ${ }^{a, b}$ Yibing $L^{b}$ and Jin-Chong Tan ${ }^{a, *}$ \\ The structural flexibility of a topical zeolitic imidazolate framework with sodalite topology, termed ZIF-8, has been elucidated \\ through liquid intrusion under moderate pressures (i.e. tens of $\mathrm{MPa}$ ). By tracking the evolution of water intrusion pressure in \\ cyclic conditions, we interrogate the role of gate-opening mechanism controlling the size variation of ZIF-8's pore channels. \\ Interestingly, we demonstrate that its channel deformation is recoverable through structural relaxation over time, hence \\ revealing the viscoelastic mechanical response in ZIF-8. We propose a simple approach employing a glycerol-water solution \\ mixture, which can significantly enhance the sensitivity of intrusion pressure for the detection of structural deformation in \\ ZIF-8. By leveraging the time-dependent gate-opening phenomenon in ZIF-8, we achieved a notable improve-ment (50\%) in \\ energy dissipation during multicycle mechanical deformation experiments.
}

\section{Introduction}

Zeolitic imidazolate frameworks (ZIFs) are a promising subfamily of microporous metal-organic frameworks (MOFs), ${ }^{1,2}$ with a zeolitic network topology assembled from tetrahedral metal centers $\left(\mathrm{MN}_{4}, \mathrm{M}=\right.$ metal ions such as $\mathrm{Zn}^{2+}$ or $\left.\mathrm{Co}^{2+}\right)$ bridged by imidazolate-based linkers. ${ }^{3}$ Notably, many ZIF structures exhibit large pore cavities, but interconnected by narrow channels (i.e. window apertures) which allow size-selective separations. ${ }^{4}$ Further to the tunable structure and functionality of ZIFs, they are advantageous over other MOFs due to enhanced chemical/thermal stability which is a typical feature of conventional zeolites. ${ }^{5}$ From the mechanical viewpoint, however, the open-framework structure of ZIFs are not as rigid as zeolites. ${ }^{6,7}$ Indeed their structural flexibility has drawn considerable attention since it may strongly affect their engineering performance ${ }^{8,9}$ in applications such as gas separations, sensing, guest storage and delivery. ${ }^{10-12}$

$\mathrm{ZIF}-8\left[\mathrm{Zn}(\mathrm{mIM})_{2}, \mathrm{mIM}=2\right.$-methylimidazolate $]$ is amongst the most investigated ZIFs due to its ease of synthesis coupled with large surface area and excellent chemical stability. ${ }^{11} \mathrm{ZIF}-8$ has a sodalite (SOD) topology with relatively narrow channels comprising 6-membered rings (window size $\sim 3.40 \AA$ ) connecting larger internal pores $(\sim 11.6 \AA ̊ \AA) .{ }^{3}$ However, 'over-sized' molecules (such as methane, $3.8 \AA$ and nitrogen, $3.64 \AA \AA^{13,14}$ can pass through these narrow channels, unveiling its structural flexibility attributed to the 'gate-opening' mechanism of ZIF-8. Gate opening in ZIF-8 involves a rotational swing effect of the labile imidazolate linkers, ${ }^{15}$ which can be triggered by either guest adsorption, ${ }^{16}$ a mechanical pressure, ${ }^{17}$ or an external electrical field. ${ }^{18}$ Unconventional two-step isotherm observed in nitrogen adsorption at $77 \mathrm{~K}$ reveals that a low gas pressure ( 0.2 and $2 \mathrm{kPa}$ ) yields gate opening upon guest uptake. ${ }^{16}$

High-pressure diamond-anvil-cell compression (DAC) experiments have been conducted to study the structural and chemical changes in ZIF-8 up to $4 \mathrm{GPa}$, by using different pressure-transmitting media (PTM). ${ }^{19}$ Using a small molecular sized penetrating (hydrophilic) liquid such as methanol and ethanol, there will be a reversible phase transition at $1.47 \mathrm{GPa}$; the high-pressure phase reorients the imidazolate linkers via gate-opening mechanism, which increases the size of the narrow channels connecting the pores. ${ }^{17}$ While if a non- penetrating fluid (e.g. Fluorinert) is used as PTM or ZIF-8 is compressed without any PTM, irreversible structural transition and amorphization will occur as the pressure goes beyond 0.34 GPa. ${ }^{20}$ However, another study with no PTM, utilizing in situ FTIR spectroscopy instead of X-ray diffraction, reported that ZIF-8 can be compressed reversibly within $1.6 \mathrm{GPa}$ and further compression to $39 \mathrm{GPa}$ will amorphize the structure. ${ }^{21}$

Despite all the foregoing efforts concerning the high pressure ( $\sim \mathrm{GPa}$ ) behavior of ZIF-8, to the best of our knowledge its structural response under a moderate liquid intrusion pressure ( $\mathrm{MPa})$ has not yet been addressed. Moderate pressures of the order of several to tens of MPa ( 10-100 bar) is central to engineering applications, for example in mechanical energy storage ${ }^{22,23}$ and energy mitigation systems, ${ }^{24,25}$ and for novel vehicle damping devices. ${ }^{26-28}$ With a bulk modulus of 7.75 GPa for ZIF- $8,{ }^{7}$ the structural distortion upon moderate pressure intrusion might be small (e.g., tens of MPa only creates a volumetric change of around $1 \%$ ), thus giving us the unique opportunity to explore whether MPa pressure can be used to modify the flexible framework structure and molecular scale pore configurations in ZIF-8.

In this study, we propose a liquid-intrusion approach to study the variation of ZIF-8's pore channels when subject to a moderate level of hydrostatic pressure in the MPa range. Instead of employing in situ XRD/FTIR techniques to track structural changes, we demonstrate the use of the penetrating liquid itself as a sensitive probe to detect nanoscale variation of channel size.

\section{Results and Discussion}

\subsection{Water intrusion response under multi-cyclic loading}

According to nanofluidics, non-wetting liquid will intrude nanopores at a critical pressure at which the capillary effect is exceeded, whereby this intrusion pressure is inversely proportional to the channel size. ${ }^{29}$ Using this principle, we can characterize the pore size variation in ZIF-8 by monitoring the intrusion pressure of certain non-wetting liquids. Since ZIF-8 is inherently hydrophobic due to the presence of methyl functional groups, ${ }^{30,31}$ it is convenient to begin by using water 
as the penetrating liquid. To demonstrate this concept, we fabricated a ZIF-8/water nanofluidic system as depicted in Figure S1 in the Supporting Information (SI). ZIF-8 powder (Basolite Z1200 from BASF, XRD shown in Figure S2) and water were combined and sealed in a stainless-steel compression chamber. The pressure was induced by a $6-\mathrm{mm}$ diameter piston inserted into the chamber driven by the Instron 5582, which records force and displacement history during the loading and unloading cycles (up to 40). We applied a constant displacement rate of $0.5 \mathrm{~mm} / \mathrm{min}$, and reversed the crosshead direction at a typical peak pressure of $56 \mathrm{MPa}$.

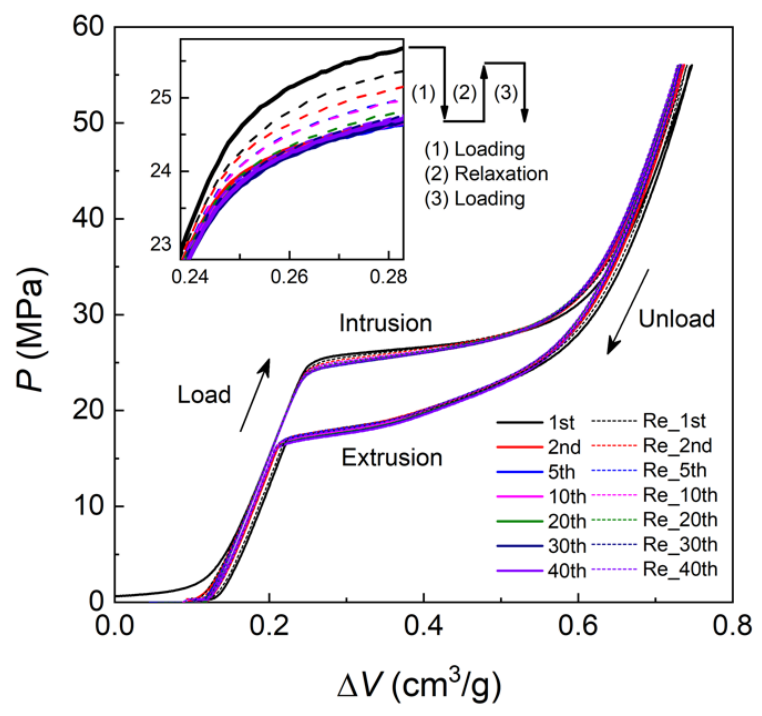

Figure 1. $P-\Delta V$ curves of water intrusion into ZIF-8, including 40 consecutive loading-unloading cycles, and followed by another 40 cycles after a 24 -hour relaxation time (dotted lines). The inset shows the transition curves at the start of water intrusion. Note that $\Delta V$ is negative due to compression.

Figure 1 shows the pressure versus volume change curves $(P-\Delta V)$ of ZIF-8, revealing its water intrusion response under the cyclic loading condition. It can be seen that, initially as the pressure increases the system volume has a linear decline attributed to elastic compression of water and ZIF-8 particles, but without any pore intrusion due to its hydrophobicity. At 25 $\mathrm{MPa}$, the capillary effect has been overcome and water molecules begin to intrude the ZIF- 8 pores. This intrusion process gives rise to a distinct plateau on the $P-\Delta V$ curve, which deviates from the elastic modulus of the system. When all the pore cavities have been occupied by water molecules, the plateau ends and another steep linear segment appears due to the compression of water and water-filled ZIF- 8 crystals. The unloading curve has an extrusion plateau: similar to the intrusion segment albeit at a relatively lower pressure down to $\sim 16 \mathrm{MPa}$, signifying that water molecules escape from ZIF-8 nanopores during decompression. It can be seen that the system recovers most of its initial volume when the applied pressure drops to zero. Though the water intrusion-extrusion phenomena elucidated above is reminiscent of the work of Patarin and co-workers, ${ }^{32,33}$ they have thus far reported the behavior of ZIF-8 for a much smaller number of deformation cycles (typically 3 ) and have not yet considered the influence of structural flexibility on time-dependent mechanical response.

Due to the excellent water extrusion performance of ZIF-8, the consecutive 40 cycles in Figure 1 exhibit almost overlapping $P-\Delta V$ curves. However, a closer examination of the data (see the inset of Figure 1) unveils a more remarkable effect. Specifically, we have detected a subtle but measureable decline of $\sim 1 \mathrm{MPa}$ in intrusion pressure upon consecutive multicycle loading. Furthermore, according to the results of another set of cyclic tests performed on the same sample after a 24-hour relaxation time, we found this systematic decline can be recovered almost fully, and then declines again upon further cyclic loading. Because there should be no change in the solid-liquid interfacial property among different cycles, we hypothesized that this systematic shift of intrusion pressure can be ascribed to the evolution of the window apertures leading into a ZIF-8 pore. That is, the narrow channels interconnecting the ZIF-8 pores have incrementally expanded in diameter due to water intrusion, and therefore allowing water molecules to intrude at a lower pressure in the subsequent cycles. This phenomenon can be explained by the gate-opening mechanism prevalent in ZIF- $8,{ }^{16,17}$ although previous experiments have not yet considered the effects of multicycle loading-unloading deformation effects. Herein, we established that there is a minor change measured in residual deformation (recoverable after structural relaxation), and a good retention of maximum volumetric change where $\Delta V \sim 0.4 \mathrm{~cm}^{3} / \mathrm{g}$. Importantly, we also confirmed that the crystalline framework of ZIF-8 remains intact after multicycle mechanical loading, evidenced by the consistent intrusion plateau length further supported by the XRD data (Figure S2 of SI) showing consistent relative Bragg peak intensities. The immersion in water does not create notable degradation of $\mathrm{ZIF}-8,^{34}$ due to its high mass ratio to water (1:4) employed in this study.

\subsection{Intrusion behavior of a glycerol-water solution mixture}

Since the variation of water intrusion pressure observed in $\S 2.1$ is relatively subtle, we turn to other probe liquid whose molecular size is comparable with that of the ZIF- 8 channels. In this way, the framework deformation can switch on and switch off the liquid intrusion and thus be easily detected. That is, the liquid molecules chosen should be slightly larger than the size of the ZIF-8 channels, so that they will intrude only after the framework has structurally deformed. To this end, glycerol is a good candidate, with its molecular size being $3.6 \AA$ in the least extended dimension, ${ }^{35}$ which is only slightly larger than ZIF-8's channel limiting diameter of $3.4 \AA$ A Notably, given that alcohol can have affinity with both water molecules and ZIF-8 framework: glycerol will promote water intrusion if they can enter the nanopores together with water molecules, while it will inhibit water intrusion if the channels are too narrow for glycerol molecules to enter. In the light of this, compared with pure water intrusion into ZIF-8 framework, we reasoned that the addition of glycerol into the liquid phase will: (i) intensify the intrusion pressure prior to any structural deformation, or (ii) diminish the intrusion pressure upon channel expansion. By 
implementing this approach, the sensitivity for the detection of ZIF-8's structural changes can then be significantly improved.

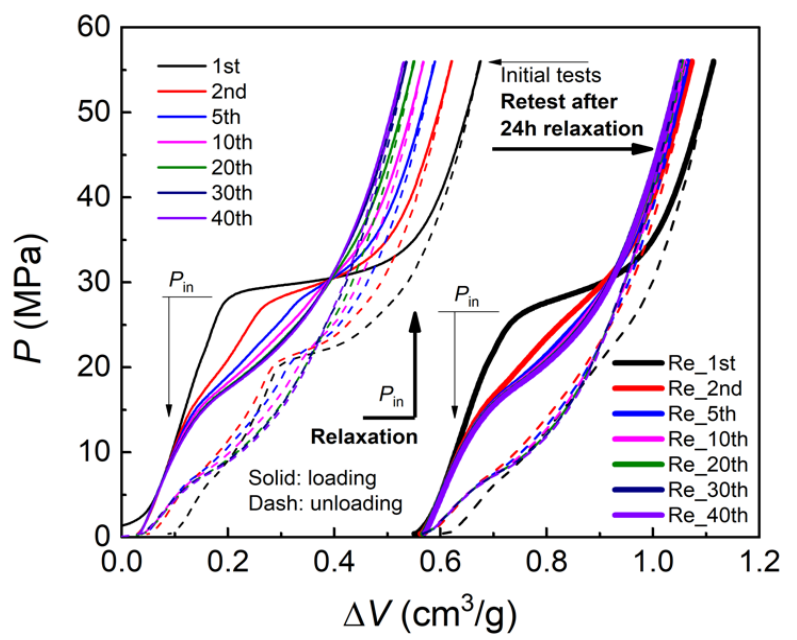

Figure 2. $P-\Delta V$ curves of glycerol-water solution into ZIF-8 framework, showing cyclic intrusion-extrusion curves of $10 \mathrm{wt} . \%$ glycerol-water system. Two sets of 40-cycle measurements were performed with a relaxation time of $24 \mathrm{~h}$ in between them. Note: Retested curves shifted horizontally for clarity. Extrusion curves are shown as dotted lines. $P_{\text {in }}$ denotes the intrusion pressure.

We have demonstrated efficacy of the above concept by systematically studying the intrusion behavior of glycerol-water solution mixture into ZIF-8. Figure 2 shows the cyclic $P-\Delta V$ curves with a glycerol concentration of $10 \mathrm{wt} . \%$. In the first cycle, the intrusion pressure has increased to $28 \mathrm{MPa}$, which is a $12 \%$ rise from the pure water intrusion value of $25 \mathrm{MPa}$. This finding indicates that glycerol is functioning as an intrusion inhibitor outside the ZIF-8 framework, due to its hydrogen bonding affinity with water molecules. Additional energy is thus required for the water molecules to dissociate themselves from glycerol-water clusters before they can enter the ZIF-8 pore channels. Furthermore, the data revealed that some liquid molecules are confined inside the ZIF- 8 channels upon loading, which may expand the channels and facilitate the entry of glycerol molecules. This is supported by our data whereby in the subsequent cycles, due to the intrusion promoting effect of glycerol, a lower plateau ( $15 \mathrm{MPa}$ ) appears and then gradually becoming dominant with consecutive loading, while the initial plateau diminishes and finally disappears. The gap between these two plateaus is $13 \mathrm{MPa}$, which is a pronounced distinction of intrusion pressure caused by framework deformation. Just like the case of pure water intrusion, the framework deformation is recoverable, which we have established by retesting the same sample after a relaxation period of 24 hours. Remarkably, we obtained the higher plateau again; this result is significant as it unambiguously proves that the ZIF-8 framework can undergo structural recovery over time. Likewise, we confirmed that subsequent multicycle reloading tests will reproduce the lower plateau again, as shown in Figure 2.
2.3 Understanding glycerol concentration effects and intrusion characteristics of related polyhydric alcohols
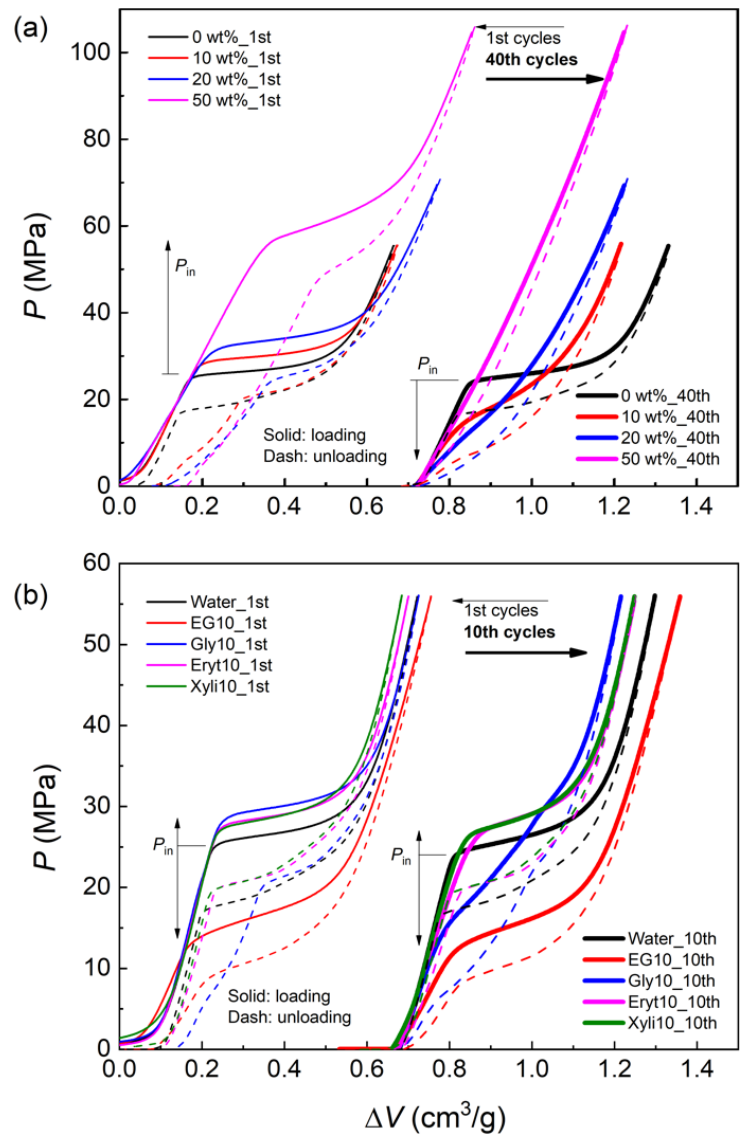

Figure 3. Multicycle intrusion-extrusion curves of glycerol-water solution into ZIF-8 framework. (a) Effects of glycerol concentration, with the higher plateau shown in the $1^{\text {st }}$ cycle and the lower plateau shown in the $40^{\text {th }}$ cycle. (b) Effects of alcohol type (at the same concentration $10 \mathrm{wt} . \%$ ), with the $1^{\text {st }}$ and $10^{\text {th }}$ cycles shown. Retested curves shifted horizontally for clarity.

For further validations, we have tested the effects of using different glycerol concentrations as shown in Figure 3(a). For the higher plateau characterized in the $1^{\text {st }}$ cycle, its intrusion pressure has increased with glycerol concentration; while for the lower plateau shown in the $40^{\text {th }}$ cycle, the intrusion pressure has decreased with glycerol concentration. This finding is consistent with the intrusion inhibiting and promoting mechanism of glycerol, both of which becoming stronger at higher concentrations. In the case of $50 \mathrm{wt} . \%$ glycerol, we cannot detect the lower plateau because its intrusion pressure has declined to zero, this finding means that: this binary liquid has become so hydrophilic it spontaneously infiltrates the ZIF-8 nanopores.

We then investigated the effects of glycerol's neighbors employing a series of polyhydric alcohols, encompassing ethylene glycol (C2), erythritol (C4) and xylitol (C5). The results are presented in Figure 3(b). As expected, due to its smaller molecular size that can be accommodated by ZIF-8 channels without distortion, ethylene glycol serves as an intrusion promoter from the very start and thus lowering the intrusion pressure to $13 \mathrm{MPa}$. In contrast, erythritol and xylitol molecules 
are simply too big to infiltrate when being forced under pressure even after water intrusion and subsequent framework deformation, therefore they act as intrusion inhibitors, resulting in higher plateaus for all the cycles. Unsurprisingly, as shown in Figure $3(\mathrm{~b})$, the lower plateau is hardly recognizable in the $10^{\text {th }}$ cycle. By comparison to our results, we note that the incorporation of other small guest molecules into ZIF-8 pore have been achieved, for example caffeine ${ }^{36}$ through a slow diffusive process under extended solution stirring (without pressure) assisted by the structural flexibility of ZIF-8. In contrast, large guest molecules such as doxorubicin are far too large to penetrate the channels and only bind to the ZIF- 8 surface, ${ }^{37}$ whereas other bulky luminescent complexes can be entrapped within the pores of ZIF-8 using in situ nanoconfinement strategy. ${ }^{38}$

\subsection{Exploring the significance of mechanical deformation rate on the intrusion response of ZIF-8}

By virtue of the proposed detection method, we are able to further explore the effects of deformation rate on mechanical properties, which is an important question but not yet explored in MOFs due to limitations of the conventional techniques. Figure 4 shows the intrusion of $10 \mathrm{wt}$.\% glycerol solution into ZIF- 8 at a displacement rate of $50 \mathrm{~mm} / \mathrm{min}$ (which is $100 \times$ greater than before). It is striking to see that, the substantially higher intrusion rate produces almost identical $P-\Delta V$ curves in each cycle, with only a relatively small drop in intrusion pressure (versus the response shown in Figure 2); this result suggests that glycerol molecules are residing outside the porous framework and thus functioning as an intrusion inhibitor even after 40 cycles.

This is an interesting outcome, in contrast to the fact that glycerol molecules can enter the nanopores upon repeated cycles by low-rate intrusion (Figure 2), here we discovered that the ZIF-8 framework deformation exhibits a strong strain-rate dependent effect. In this context, our data suggest that the channel deformation by gate-opening mechanism takes time to occur, where the channel deforms gradually upon low-rate liquid intrusion and it needs continuous application of pressure to be fully triggered. To validate this rate effect, after the above cyclic loadings, the piston was held at the peak pressure (56 $\mathrm{MPa}$ ) for 4 minutes to allow structural deformation to occur. Then in the following loading cycle (still at $50 \mathrm{~mm} / \mathrm{min}$ ), we succeeded in obtaining a lower plateau at $24 \mathrm{MPa}$ (which can recover after relaxation). Indeed, this result validated our conjecture that the gate-opening response of ZIF-8 is timedependent and associated with the gradual deformation of its structural moieties, or, on the macroscale this results in the rate-dependent 'viscoelastic' response of the ZIF-8 material.

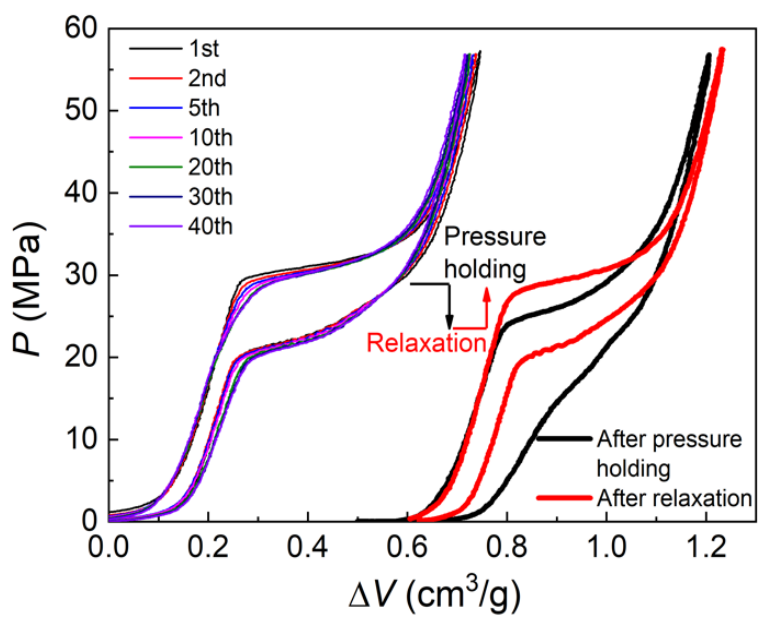

Figure 4. $P$ - $\Delta V$ curves of 10 wt.\% glycerol-water solution into ZIF-8 framework at $50 \mathrm{~mm} / \mathrm{min}$, including 40 consecutive loading cycles, one following cycle after pressure holding at $56 \mathrm{MPa}$ for $4 \mathrm{~min}$, and a final cycle tested after a $24 \mathrm{~h}$ framework relaxation.

\section{Concluding Remarks}

In summary, we have elucidated a new method to probe the structural deformation of ZIF-8 at the molecular level, the approach is applicable to other MOF materials by adopting suitable non-wetting penetrating liquids. This study provides the first evidence of ZIF-8 framework flexibility upon pressureinduced liquid intrusion, accommodated by the gate-opening mechanism. Specifically, we show external pressure at tens of MPa proves to be capable of deforming the ZIF-8 framework, but without causing detrimental framework degradation subject to multicyclic loading. Our data revealed intriguing pore channel recovery through structural relaxation. We have discovered the viscoelasticity of the ZIF-8 framework, by tracking the rate effects of its deformation under liquid intrusion conditions. Although it has been reported that the crystal size of ZIF-8 may influence the water intrusion pressure, $^{33}$ it remains to be established whether ZIF-8's framework flexibility and relaxation behavior are also size dependent subject to liquid intrusion.

It is envisaged that our findings not only will open up the field of rate-dependent MOF mechanics, ${ }^{39-41}$ but also lead to new MOF-based nanofluidic innovations. ${ }^{22,42}$ For example, highrate liquid intrusion performance is critical for applications like impact mitigators and shock absorbers afforded by nanoporous materials. To this end, we have calculated energy dissipations associated with liquid intrusion into ZIF-8 (Table S1). Remarkably, we obtained up to $\sim 50 \%$ enhancement in energy dissipation upon multicycle loading (Figure S3(c)) simply by exploiting the rate-sensitivity of ZIF-8, such time-dependent gate-opening phenomenon in MOFs warrants further investigations by using an even greater deformation strain rate. 


\section{Acknowledgements}

Y. Sun would like to thank the K.C. Wong Fellowship for funding this research. We thank Prof. Clive Siviour at Oxford University for helpful discussions concerning the mechanical response of rate-dependent materials. We are grateful to the ISIS Rutherford Appleton Laboratory, especially Dr. Gavin Stenning and Dr Marek Jura at R53 Materials Characterization Laboratory for access to the XRD facilities. We thank Chengliang $\mathrm{Xu}$ for assistance in conducting the higher rate intrusion tests.

Author Contributions: Y.S. and J.C.T. conceived the project. Y.S. designed and performed the experiments. Y.S. analyzed the data with guidance from J.C.T. Y.S. and J.C.T. prepared the manuscript. All authors contributed to the discussion of results and have given approval to the final version of the manuscript.

\section{References}

1. M. D. Allendorf and V. Stavila, CrystEngComm, 2015, 17, 229-246.

2. J. C. Tan and B. Civalleri, CrystEngComm, 2015, 17, 197-198.

3. A. Phan, C. J. Doonan, F. J. Uribe-Romo, C. B. Knobler, M. O'Keeffe and O. M. Yaghi, Acc. Chem. Res., 2010, 43, 58-67.

4. R. Banerjee, A. Phan, B. Wang, C. Knobler, H. Furukawa, M. O'Keeffe and O. M. Yaghi, Science, 2008, 319, 939-943.

5. K. S. Park, Z. Ni, A. P. Cote, J. Y. Choi, R. D. Huang, F. J. UribeRomo, H. K. Chae, M. O'Keeffe and O. M. Yaghi, Proc. Natl. Acad. Sci. USA, 2006, 103, 10186-10191.

6. J. C. Tan, T. D. Bennett and A. K. Cheetham, Proc. Natl. Acad. Sci. USA, 2010, 107, 9938-9943.

7. J. C. Tan, B. Civalleri, C. C. Lin, L. Valenzano, R. Galvelis, P. F. Chen, T. D. Bennett, C. Mellot-Draznieks, C. M. Zicovich-Wilson and A. K. Cheetham, Phys. Rev. Lett., 2012, 108, 095502.

8. J. C. Tan and A. K. Cheetham, Chem. Soc. Rev., 2011, 40, 10591080.

9. N. C. Burtch, J. Heinen, T. D. Bennett, D. Dubbeldam and M. D. Allendorf, Adv. Mater., 2017, DOI: 10.1002/adma.201704124, 1704124.

10. L. Diestel, N. Y. Wang, B. Schwiedland, F. Steinbach, U. Giese and J. Caro, J. Membr. Sci., 2015, 492, 181-186.

11. C. Zhang and W. J. Koros, J. Phys. Chem. Lett., 2015, 6, 3841-3849.

12. C. Gucuyener, J. van den Bergh, J. Gascon and F. Kapteijn, J. Am. Chem. Soc., 2010, 132, 17704-17706.

13. W. Zhou, H. Wu, M. R. Hartman and T. Yildirim, J. Phys. Chem. C, 2007, 111, 16131-16137.

14. Q. Song, S. K. Nataraj, M. V. Roussenova, J. C. Tan, D. J. Hughes, W. Li, P. Bourgoin, M. A. Alam, A. K. Cheetham, S. A. Al-Muhtaseb and E. Sivaniah, Energy Environ. Sci., 2012, 5, 8359-8369.

15. M. R. Ryder, B. Civalleri, T. D. Bennett, S. Henke, S. Rudić, G. Cinque, F. Fernandez-Alonso and J. C. Tan, Phys. Rev. Lett., 2014, 113, 215502.

16. D. Fairen-Jimenez, S. A. Moggach, M. T. Wharmby, P. A. Wright, S. Parsons and T. Duren, J. Am. Chem. Soc., 2011, 133, 8900-8902.

17. S. A. Moggach, T. D. Bennett and A. K. Cheetham, Angew. Chem. Int. Ed., 2009, 48, 7087-7089.

18. A. Knebel, B. Geppert, K. Volgmann, D. I. Kolokolov, A. G. Stepanov, J. Twiefel, P. Heitjans, D. Volkmer and J. Caro, Science, 2017, 358, 347-351.

19. J. Im, N. Yim, J. Kim, T. Vogt and Y. Lee, J. Am. Chem. Soc., 2016, 138, 11477-11480.
20. K. W. Chapman, G. J. Halder and P. J. Chupas, J. Am. Chem. Soc., 2009, 131, 17546-17547.

21. Y. Hu, H. Kazemian, S. Rohani, Y. N. Huang and Y. Song, Chem. Commun., 2011, 47, 12694-12696.

22. G. Fraux, F. X. Coudert, A. Boutin and A. H. Fuchs, Chem. Soc. Rev., 2017, 46, 7421-7437.

23. F. B. Surani, X. Kong, D. B. Panchal and Y. Qiao, Appl. Phys. Lett., 2005, 87, 163111.

24. Y. Sun, Z. Guo, J. Xu, X. Xu, C. Liu and Y. Li, Mater. Des., 2015, 66, 545-551.

25. Y. Sun, X. Xu, C. Xu, Y. Qiao and Y. Li, Sci. Rep., 2016, 6, 26694.

26. P. G. Yot, Z. Boudene, J. Macia, D. Granier, L. Vanduyfhuys, T. Verstraelen, V. Van Speybroeck, T. Devic, C. Serre, G. Ferey, N. Stock and G. Maurin, Chem. Commun., 2014, 50, 9462-9464.

27. C. V. Suciu and K. Yaguchi, Experimental Mechanics, 2008, 49, 383-393.

28. C. V. Suciu, T. Iwatsubo and S. Deki, J. Colloid Interface Sci., 2003, 259, 62-80.

29. Y. Qiao, L. Liu and X. Chen, Nano Lett., 2009, 9, 984-988.

30. K. Zhang, R. P. Lively, M. E. Dose, A. J. Brown, C. Zhang, J. Chung, S. Nair, W. J. Koros and R. R. Chance, Chem. Commun., 2013, 49, 3245-3247.

31. A. U. Ortiz, A. P. Freitas, A. Boutin, A. H. Fuchs and F. X. Coudert, Phys. Chem. Chem. Phys., 2014, 16, 9940-9949.

32. G. Ortiz, H. Nouali, C. Marichal, G. Chaplais and J. Patarin, J. Phys. Chem. C, 2014, 118, 7321-7328.

33. I. Khay, G. Chaplais, H. Nouali, C. Marichal and J. Patarin, RSC Adv., 2015, 5, 31514-31518.

34. H. Zhang, D. Liu, Y. Yao, B. Zhang and Y. S. Lin, J. Membr. Sci., 2015, 485, 103-111.

35. L. Y. Chen, J. Struct. Biol., 2013, 181, 71-76.

36. N. Liedana, A. Galve, C. Rubio, C. Tellez and J. Coronas, ACS Appl. Mater. Interfaces, 2012, 4, 5016-5021.

37. I. B. Vasconcelos, T. G. da Silva, G. C. G. Militao, T. A. Soares, N. M. Rodrigues, M. O. Rodrigues, N. B. da Costa, R. O. Freire and S. A. Junior, RSC Adv., 2012, 2, 9437-9442.

38. A. K. Chaudhari, M. R. Ryder and J. C. Tan, Nanoscale, 2016, 8, 6851-6859.

39. Z. Su, W. L. Shaw, Y. R. Miao, S. You, D. D. Dlott and K. S. Suslick, J. Am. Chem. Soc., 2017, 139, 4619-4622.

40. K. Banlusan and A. Strachan, J. Phys. Chem. C, 2016, 120, 1246312471.

41. Z. Zeng and J. C. Tan, ACS Appl. Mater. Interfaces, 2017, 9, 3983939854.

42. P. G. Yot, L. Vanduyfhuys, E. Alvarez, J. Rodriguez, J.-P. Itié, P. Fabry, N. Guillou, T. Devic, I. Beurroies, P. L. Llewellyn, V. Van Speybroeck, C. Serre and G. Maurin, Chem. Sci., 2016, 7, 446-450. 